\title{
Measurement of large angle muon flux in GRAPES-3 experiment using triggerless DAQ system
}

\author{
B. Hariharan* on behalf of the GRAPES-3 Collaboration \\ (a complete list of authors can be found at the end of the proceedings) \\ E-mail: 89hariharan@gmail.com
}

\begin{abstract}
The large area muon telescope of GRAPES-3 has been operating continuously for more than two decades with a DAQ which has several limitations. At present, this DAQ is in the process of being upgraded with a FPGA based system. The new DAQ system is designed to be triggerless and capable of recording every hit from the 3712 proportional counters along with a time-stamp ( $10 \mathrm{~ns}$ resolution) which has significantly expanded the physics horizon of the experiment. This triggerless feature allows the detection of muons beyond the nominal zenith range of the current system $\left(\theta<45^{\circ}\right)$. The upgraded DAQ system has been deployed for $25 \%$ of the telescope. An offline software trigger has been developed for the reconstruction of muon tracks by using the timing and pulse height information of each hit in the raw data. For the first time the muons are reconstructed in the entire zenith angle range. The extensive air showers (EAS) at large angles can be studied through the muon component. We present measurements of the flux of the large angle muons and their correlation with EAS triggers.
\end{abstract}

$37^{\text {th }}$ International Cosmic Ray Conference (ICRC 2021)

July 12th - 23rd, 2021

Online - Berlin, Germany

*Presenter 


\section{Introduction}

In the recent past the GRAPES-3 muon telescope (G3MT) has demonstrated its capabilities with some of the ground breaking discoveries using the measurements on angular distribution of muons recorded with high statistics [1,2]. The real-time measurement of arrival direction of muon is achieved by reconstructing the track using proportional counter (PRC) hits. The G3MT is being operated for nearly two decades with the DAQ system designed during that era. Due to limited CPU and storage resources muons were reconstructed in real-time and only the muon rate as a function of arrival direction was stored. The online reconstructed muons are binned into $169(13 \times 13)$ arrival directions in $\theta-\phi$ space and stored the integral rate in each angular bin at every 10 seconds with a dead time of about $12 \%$ due to slow electronic interfaces. Thunderstorm phenomenon in the atmosphere induces sudden changes in the flux of muons. However, such measurements at shorter time scale are limited by the slow electronics. Triggerless muon DAQ system (TM-DAQ) overcomes this problem and provides dead time free measurements of muon flux. Furthermore, same DAQ stream enables independent study of large angle muons (not coupled to extensive air shower (EAS) trigger), search for delayed particles in EASs and other exotic physics. The electromagnetic component of EASs does not arrive at surface of the Earth at large angles. Thus, it is only the muon component through which EAS can be studied at large angles. The large angle muons are expected to have higher energy at production since they have to pass through a greater thickness of Earth's atmosphere, thus, they sample higher energy cosmic rays (CRs) as compared to those arriving at lower zenith angle region. Old G3MT DAQ system can record the rate of muons passing through all the 4 layers of module only up to the zenith angle of $\theta<45^{\circ}$, whereas, the TM-DAQ does not have any such limitations and it can reconstruct large angle muons passing through even one layer of the module with sufficiently large number of hits.

FPGA based TM-DAQ enables to record raw data of each PRC hit (pulse width and precise timing of $10 \mathrm{~ns}$ resolution) which is required for offline reconstruction of muons in the data. It is to be noted that response time of PRC is about $\sim 400 \mathrm{~ns}$. The hardware trigger is generated in FPGA by taking layer OR signal from each layer and then taking AND of these signals from all four layer within $3 \mu$ s (4-Fold trigger). The offline software muon trigger algorithm is developed for reconstruction of muons using only PRC hits data. The recorded data mainly contains the width and time of each PRC hit, all types of triggers, the 64-bit clock counter etc. The TM-DAQ is almost dead time free. The software trigger generation algorithm is demonstrated to have reasonably good agreement with the 4-Fold hardware triggers. With that agreement, the software trigger algorithm is extended to identify large angle muons that are not passing through all the layers of the module.

\section{The GRAPES-3 muon telescope}

The basic building block of the G3MT is a PRC which is built using a $6 \mathrm{~m}$ long mild steel tube (wall thickness of $2.3 \mathrm{~mm}$ ) having square cross section of $10 \mathrm{~cm} \times 10 \mathrm{~cm}$ filled with a mixture of $90 \%$ argon and 10\% methane gas (P10 gas). At the center of the tube, a $100 \mu \mathrm{m}$ thick tungsten wire is laid and electrically isolated from the body by using hermetic seal. The PRC is operated at 3000 Volts providing adequate gain to efficiently detect pulses due to passage of charged particles. The G3MT consists of 16 independent muon modules each having four layers of PRCs. A carpet of 

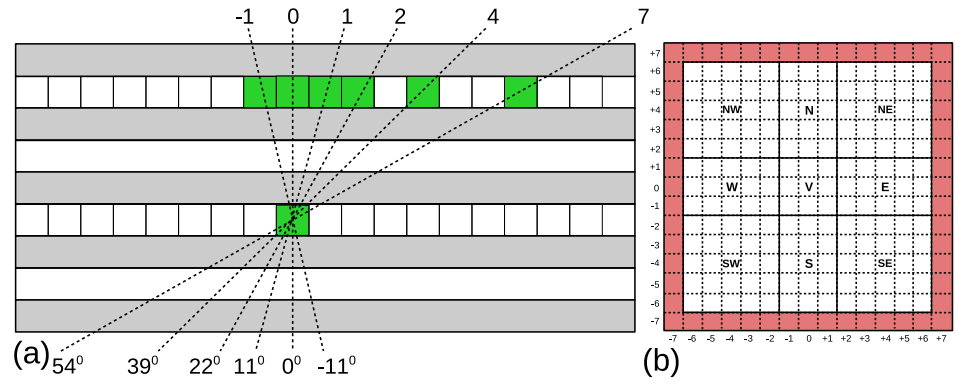

Figure 1: Representation of (a) muon direction reconstruction in a single projection using PRC hits and (b) grid of reconstructed $15 \times 15=225$ directions, the inner grid of $13 \times 13=169$ directions are shown by dashed lines, the number of actual usable directions for physics studies.

58 PRCs is laid in a tightly packed fashion in each layer. Alternate layers are arranged orthogonal to each other to get the stereoscopic view of the trajectory. Successive layers are sandwiched by $15 \mathrm{~cm}$ thick concrete slabs. The angular resolution of the trajectory gradually improves with the incident zenith angle. Existing real-time trigger system covers a solid angle of $2.3 \mathrm{sr}\left(\theta \leq 45^{\circ}\right)$ in the upper hemisphere (Figure 1) with a mean angular resolution of $4^{\circ}$. Above the top layer, a $2 \mathrm{~m}$ thick concrete slabs are placed in the inverted pyramidal shape. The overburden absorbs soft components ( $e^{ \pm}$and $\gamma$-rays) and muons below $1 \mathrm{GeV}$. The concrete shielding is deployed to provide coverage up to $45^{\circ}$ [3]. The G3MT with sixteen modules is being operated for nearly two decades recording the data with an integrated muon trigger rate of $50 \mathrm{kHz}$ providing a large statistics of 4 billion muons per day binned in $13 \times 13$ direction in $\theta-\phi$ space.

\section{Triggerless muon DAQ system (TM-DAQ)}

Existing muon DAQ system is quite old and difficult to maintain. Also, it has several inherent limitations to expand the physics scope of the detector. In view of this, the new TM-DAQ for G3MT has been designed which is not only robust and compact but also provides excellent capability to explore the physics beyond standard model. It is built using FPGA based boards designed for ALICE experiment at CERN [4] which were decommissioned from the experiment as a part of upgrade program. The boards are equipped with a XILINX Virtex4 FPGA having large memory and various communication modules such as Ethernet, JTAG, TAGNET, and PCI. The board provides 158 programmable I/O pins which can be used for any application. Digital signals from either two even layers or two odd layers (58 channels/layer) are provided as the input to the board. Two ALICE boards are used to readout data from an entire muon module. The clock to the FPGA is provided by the onboard $100 \mathrm{MHz}$ and $50 \mathrm{MHz}$ highly stable crystal oscillators, thus providing a timing resolution of $10 \mathrm{~ns}$ for the time stamp of PRC hit. External global positioning system (GPS) trigger at $1 \mathrm{~Hz}$ is provided as one of the input to the board using which the on board clock is calibrated at every second. The real time clock (RTC) onboard is synchronised with the GPS trigger at the beginning of every calendar second. The EAS trigger generated by the scintillator array and time calibration trigger (TCT) is also provided as one of the inputs to ALICE board. The Layer-OR trigger generated for each layer on the board are shared with other board through $\mathrm{I} / \mathrm{O}$ pins. The Layer-OR signals are used to generate triggers of various types such as 4-Fold (AND of 


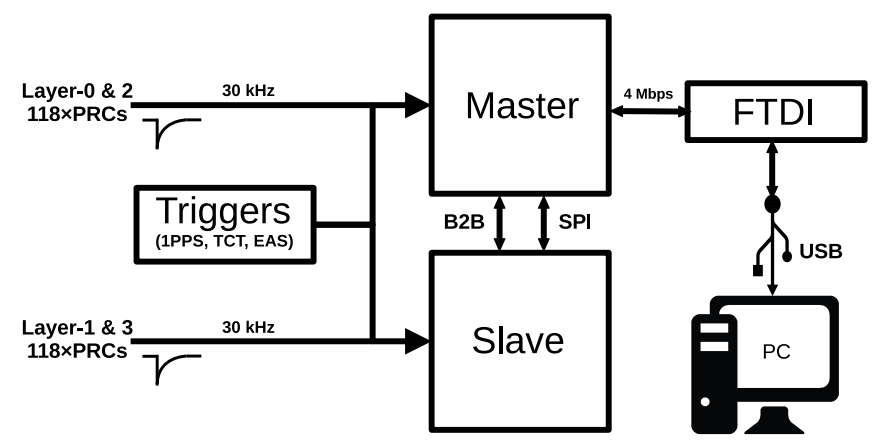

Figure 2: Block diagram of the new triggerless muon DAQ system for muon module.

Layer-OR trigger from all the four layers within a timing window of $3 \mu \mathrm{s}$ ), 3-Fold and 2-Fold. The TM-DAQ records the width and arrival time of each pulse due to PRC hits as well as all types of triggers. The pulse width of PRC hit is a measure of energy loss of charged particle passing through it. PRC being the gaseous detector, its timing response is typically about $400 \mathrm{~ns}$; much coarser than the measurement resolution of $10 \mathrm{~ns}$. Typical hit rate of layer is about $15 \mathrm{kHz}$, thus, the pulse width and timing of typically 60k PRC hits are recorded every second. In addition to this, for monitoring purpose, we also record the counting rate of each PRC, varieties of triggers, temperature at three different locations etc. The gain of each PRC is monitored continuously by building pulse width histogram using the pulse width data. A simplified block diagram of TM-DAQ is shown in Figure 2. New TM-DAQ has more advanced features than the old DAQ system. In the old system the pulse width and timing data is recorded only when there is EAS trigger with much coarser timing and pulse width resolution (333 ns). Pulse width spectrum in the old system is recorded for duration of 1000 seconds roughly at every 18 hours.

The TM-DAQ is designed for each module and operates independent of the same for other modules. The system consists of two ALICE-FPGA boards, referred as master and slave boards mounted on a indigenously designed common interface board (Ref. Figure 2). The master and slave board manages the PRC signals from two even and two odd layers respectively. These boards communicate with each other through SPI protocol. The board-to-board (B2B) communication is used for sharing of signals among the interface boards for various layer coincidence trigger logic generation. Data collected by the slave board is transferred to master via SPI and written to the PC every second using the indigenously designed USB interface. Temperature sensors are installed on top of $100 \mathrm{MHz}$ and $50 \mathrm{MHz}$ crystals and on the surface of the board to monitor and characterise the drift in the clock. The temperature is measured at every $250 \mathrm{~ms}$. Additionally the clock counters for $100 \mathrm{MHz}$ and $50 \mathrm{MHz}$ clocks are recorded every second as 64-bit information. The clock counter recorded at GPS trigger arriving every second provides the time stamp with a resolution of $10 \mathrm{~ns}$. The dead time of the new DAQ is extremely small $(<0.001 \%)$ as compared to the older system $(\sim 12 \%)$. At present the new system records input signals at the rate of about $60 \mathrm{kHz}$ for each module. It transfers at the data rate of $\sim 4 \mathrm{Mbps}$. In spite of the high data volume (45 Giga-Byte/day) recorded everyday the dead time of the new system is negligibly small $(\sim 0.001 \%)$. More details about the design of TM-DAQ can be found in another presentation [5]. 


\section{Reconstruction of muon tracks}

In the old DAQ system, the muon tracks are reconstructed online for each module by using the PRC hits in each layer. The reconstruction of muon direction is achieved by finding direction in each of the two projected planes, namely even (using PRC hits in layers 0 and 2) and odd (using layers 1 and 3 hits). On generation of 4-Fold trigger, PRC hits with healthy signal are identified using which clusters are formed in each layer. Triggers with several clusters in each layer are rejected. The spatial separation of the cluster in top layer is estimated with respect to the bottom layer as shown in Figure 1a. A 2D histogram with spatial separation between -7 to +7 in each projection is populated during data taking and stored in PC at every 10 second. The last bin is used as an overflow (spatial separation $\geq 7$ ). The spatial separations represents well defined angular bins of different bin sizes. Incident angle of muon is obtained using spatial separation in both the projections. Since, the extreme bins are used as overflows, the outer most bins are excluded and the remaining data, hence, data from 169 angular bins are used for physics studies.

In the new TM-DAQ, the pulse width and timing (PWT) data is latched by FPGA immediately after the end of pulse in PRC (or any trigger) and then the PWT data is pushed to the DDR memory. It is to be noted that the pulse arriving later but having a shorter width may get recorded first compared to an early pulse with a larger pulse width. In total, $\sim 68 \mathrm{k}$ PWT packets (includes PRC hits and various triggers) are recorded every second in PC. The PWT packet has a length of 64 bits, consisting of 16 bit for channel address, 16 bit for pulse width, and 32 bit for timing. The pulse width of PRC hit is essentially the output of logarithmic amplifier with a fixed decay constant. Thus, the pulse height of the signal representing the energy loss of charged particle in the PRC can be calculated using the pulse width and decay constant of the amplifier. To reduce the data volume, the arrival time of PRC hit in a PWT packet consists of only 32-bit LSB of 64-bit $100 \mathrm{MHz}$ clock counter. Due to this, the arrival time resets approximately at every 42 seconds. Hence, the complete 64-bit time stamp for each hit is regenerated with the help of actual 64-bit clock count of $100 \mathrm{MHz}$ recorded at the beginning of current and of previous events. The pulse width and timing are recorded with a resolution of $10 \mathrm{~ns}$. Muons in the data can be reconstructed by identifying PRC hits associated with the 4-Fold trigger, referred as hardware trigger approach or by clustering only PRC hits (without using trigger information) using their time stamp, referred as software trigger approach. In following sub-sections, we describe how muons are constructed using each of the approaches.

\subsection{Identification of tracks using hardware trigger}

For offline reconstruction of muon, the PRC hits associated with the muon are identified in following steps: (a) 4-Fold triggers generated in both boards are paired by requiring their time stamp to match within a windows of $1 \mu \mathrm{s}$ (b) for matched 4-Fold trigger, the associated PRC hits are identified by matching PRC hit time with that of 4-Fold trigger within a time window of $3 \mu$ s. PRC hits with small pulse width and large time difference w.r.t. 4-Fold trigger time are rejected. The clusters in each layer are built using good quality PRC hits associated with the trigger. Events with only one cluster in each of the four layers are considered for reconstruction. Straight line tracks are fitted in each projection using the position of selected PRC hits in the module. The zenith and 
azimuth angles of the incident muon are obtained using the fitted slopes $\left(\mathrm{S}_{e}\right.$ and $\left.\mathrm{S}_{o}\right)$ in both the projected planes using the equations $\theta_{e, o}=\arctan \left(\mathrm{S}_{e, o}\right), \theta=\arctan \left(\sqrt{S_{e}^{2}+S_{o}^{2}}\right)$, and $\phi=\arctan \left(\mathrm{S}_{o} / \mathrm{S}_{e}\right)$.

The hardware trigger approach is based on the 4-Fold coincidence requiring muons to pass through all the 4 layers, thereby, significantly reducing the aperture of the detector with increasing zenith angle. Hence, the true potential of the TM-DAQ remains under utilised to reconstruct muons at large angles that are not passing through all the four layers. So, algorithm of muon reconstruction using only the PRC hit data (without the aid of hardware triggers) is developed. This approach does not necessarily needs the muon to pass through all the layers and it will be referred as software trigger in further discussion.

\subsection{Identification of tracks using software trigger}

Software trigger is primarily developed for identifying large angle muons which are not going through all the layers of the module. Timing information of PRC hits with sufficiently higher pulse width is used for generating the software muon trigger in following manner:

1. $100 \mathrm{MHz}$ clock is calibrated using the external GPS trigger received at every calendar second. The calibration at every second removes the effect of clock drift due to change in the ambient temperature.

2. An absolute time with resolution of $10 \mathrm{~ns}$ of each hit is obtained using the clock calibration, 64-bit clock counter recorded at every second and the 32 bit clock counter recorded for each hit. It is to be noted that while 32 bit clock counters resets at every 42 seconds, the 64-bit counter would take more than 5000 years to reset!

3. PRC hits from even and odd layers are merged and sorted in ascending order of time.

4. Clusters of two successive PRC hits are formed with the time difference of $\Delta t<1.1 \mu$ s between them. The value of $\Delta \mathrm{t}$ is obtained by optimising the matching between the software and the hardware triggers.

The genuineness of the software 4-Fold trigger may be verified with the hardware recorded 4-Fold triggers available within the PWT data bank itself. Both triggers should have a time delay less than $1 \mu$ s to qualify as a match. A maximum of $97.9 \%$ of match between software and hardware 4-Fold triggers are achieved when $1.1 \mu$ s successive delay of PRC hits is used. The total match is further increased to $99.6 \%$ when the software 4-Fold triggers are also matched with hardware recorded 3-Fold triggers. This may be attributed to the missing 4-Fold triggers which are recorded as 3-Fold triggers in the hardware. With the reasonably good agreement achieved between software and hardware triggers the algorithm can be further used to explore the large angle muon tracks.

\section{Detection of large angle muons}

Software muon trigger is used to identify and reconstruct large angle muons that are going through either all the layers or some of the layers of the detector. Figure 3 shows a typical example of large angle muon that passes through all the four layers. In the old system this muon would have 


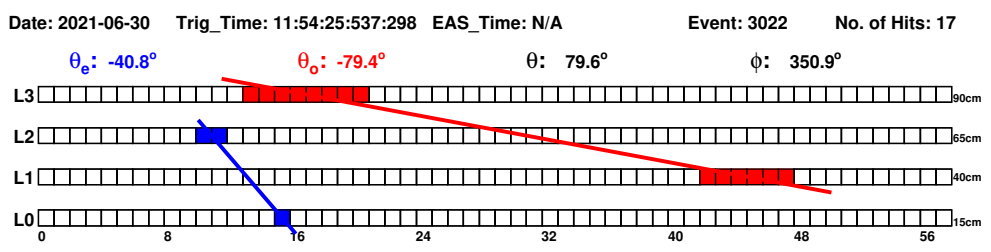

Figure 3: A sample of large angle muon passing through all four layers.

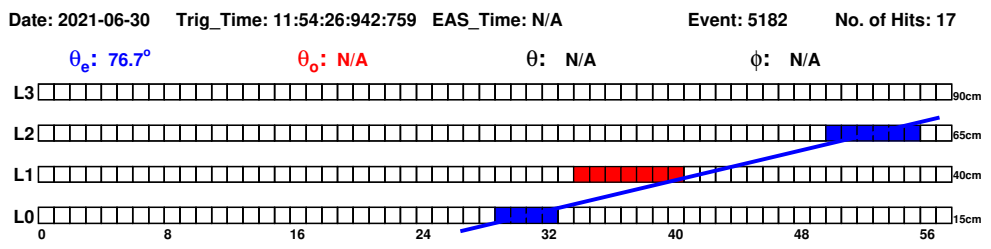

Figure 4: A sample of large angle muon passing sidewise through bottom three layers.

been recorded in the overflow bin as shown in Figure 1a. In an another example shown in Figure 4, it can be seen that the muon enters sidewise of G3MT and passes through the bottom three layers. Such topologies are not recorded in the old system. Also, there is a possibility that the same muon may even pass through one of its neighbouring modules. We plan to do full reconstruction of such topologies using data from adjacent modules. It is extremely important to ensure synchronisation of timing hits across the modules. This is achieved using the TCT obtained from the same source and fed to even and odd boards of all the modules simultaneously at every $100 \mathrm{~ms}$. These trigger hits in all the modules would be aligned and then used to reconstruct such topologies.

For a qualitative comparison, a small set of Monte Carlo dataset is generated using CORSIKA v76900 using SIBYLL and GHEISHA hadronic event generators. Since, CRs are predominantly composed of protons, the simulation is carried out with only protons in the energy range of $10^{10}$ $10^{13} \mathrm{eV}$. One million proton initiated EAS are simulated in the zenith ranging from 0 to $85^{\circ}$. Figure 5 shows a normalised zenith angle $\left(60^{\circ}<\theta<85^{\circ}\right)$ distribution of muons obtained from data and Monte Carlo simulation. The comparison shows a reasonably good agreement between them. The muons
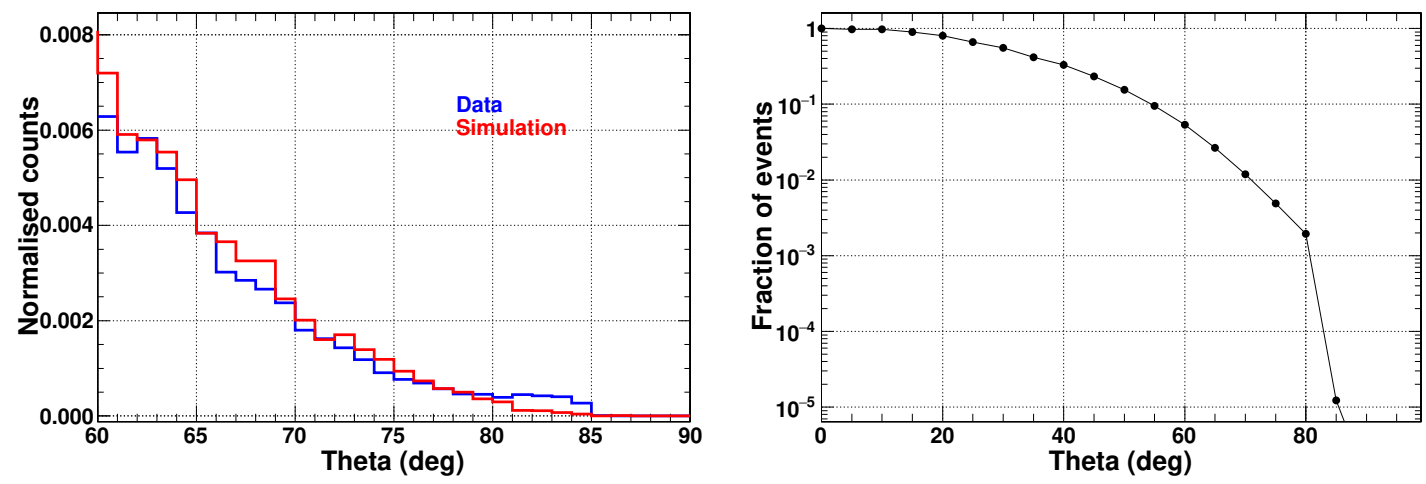

Figure 5: Comparison of normalised zenith distri- Figure 6: Profile shows integral distribution of fracbutions for $\theta \geq 60^{\circ}$ shows a reasonably good agree- tion of events as a function of zenith angle for the ment between data and simulation. muons passing through all four layers. 
that pass through all the four layers from the data are collated for this purpose. The angular accuracy of reconstruction improves with zenith angle (Ref. Figure 1a), hence, the comparison of the shape of angular distribution is made at higher zenith angle in Figure 5. Similarly, the Figure 6 shows the profile of integral fraction of muons as a function of zenith angle. It can be seen that there is a good fraction $\left(\sim 5 \%\right.$ for $\left.\theta>60^{\circ}\right)$ of large angle muons passing through all the four layers that have good angular resolution. This study would be further extended to fully reconstruct large angle muon tracks passing through the neighbouring modules (Figure 4).

\section{Discussions}

New FPGA based TM-DAQ for the G3MT has been deployed for 25\% of the muon detector and it has demonstrated all its salient features as per the design expectation. The system is practically dead time free compared to the dead time of $12 \%$ in the old system. Timing of each PRC hit is recorded with a resolution of $10 \mathrm{~ns}$. The software trigger and reconstruction algorithm has been developed to reconstruct muon trajectories. It has been shown to have a good match with the muons reconstructed using 4-Fold hardware triggers and Monte Carlo predictions (shape). Unlike old system, the TM-DAQ has the ability to identify and reconstruct muons in the entire range of zenith angles. The global time calibration trigger system referred as TCT allows to synchronise timing of all the subsystems such as 16 muon modules, EAS array, NaI detector etc. With all these features, the upgraded G3MT would allow to study the EAS in the entire zenith range and also explore physics beyond standard model.

\section{Acknowledgments}

We thank D.B. Arjunan, A.S. Bosco, V. Jeyakumar, S.Kingston, S. Murugapandian, S. Pandurangan, B. Rajesh, V. Santhoshkumar, and C. Shobana for their efforts in maintaining the GRAPES-3 experiment. The TM-DAQ was developed with the support of FPGA based boards provided by ALICE collaboration.

\section{References}

[1] P.K. Mohanty et al., Phys. Rev. Lett. 117, 171101 (2016).

[2] B. Hariharan et al., Phys. Rev. Lett. 122, 105101 (2019).

[3] Y. Hayashi et al., Nucl. Instrum. Methods A 545, 643 (2005).

[4] http://publikationen.ub.uni-frankfurt.de/frontdoor/index/index/year/2018/docId/45522

[5] A. Jain et al., Proceedings of Science PoS(ICRC2021)257. 


\section{Full Authors List: GRAPES-3 Collaboration}

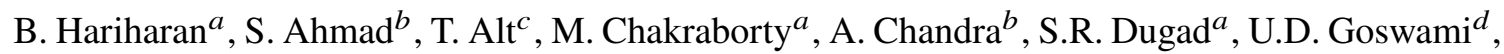
S.K. Gupta ${ }^{a}$, Y. Hayashi ${ }^{e}$, P. Jagadeesan ${ }^{a}$, A. Jain ${ }^{a}$, P. Jain ${ }^{f}$, S. Kawakami ${ }^{e}$, H. Kojima ${ }^{g}$, V. Lindenstruth ${ }^{h}$, S. Mahapatra ${ }^{i}$, K. Manjunath ${ }^{a}$, P.K. Mohanty ${ }^{a}$, R. Moharana ${ }^{j}$, Y. Muraki ${ }^{k}$, P.K. Nayak ${ }^{a}$, T. Nonaka ${ }^{l}$, A. Oshima ${ }^{g}$, B.P. Pant ${ }^{j}$, D. Pattanaik ${ }^{a, i}$, G.S. Pradhan ${ }^{m}$, P.S. Rakshe ${ }^{a}$, M. Rameez ${ }^{a}$, K. Ramesh ${ }^{a}$, L.V. Reddy ${ }^{a}$, R. Sahoo ${ }^{m}$, R. Scaria ${ }^{m}$, M.S. Shareef ${ }^{a}$, S. Shibata ${ }^{g}$, J. Soni ${ }^{f}$, R. Sureshkumar ${ }^{a}$, K. Tanaka ${ }^{n}$, F. $\operatorname{Varsi}^{f}{ }^{f}$ and M. Zuberi ${ }^{a}$.

${ }^{a}$ Tata Institute of Fundamental Research, Homi Bhabha Road, Mumbai 400005, India

${ }^{b}$ Aligarh Muslim University, Aligarh 202002, India

${ }^{c}$ Institut für Kernphysik, Johann Wolfgang Goethe-Universität Frankfurt, Frankfurt, Germany

${ }^{d}$ Dibrugarh University, Dibrugarh 786004, India

${ }^{e}$ Graduate School of Science, Osaka City University, Osaka 558-8585, Japan

${ }^{f}$ Indian Institute of Technology Kanpur, Kanpur 208016, India

${ }^{g}$ College of Engineering, Chubu University, Kasugai, Aichi 487-8501, Japan

${ }^{h}$ Frankfurt Institute for Advanced Studies, Johann Wolfgang Goethe-Universität Frankfurt, Frankfurt, Germany

${ }^{i}$ Utkal University, Bhubaneshwar 751004, India

${ }^{j}$ Indian Institute of Technology Jodhpur, Jodhpur 342037, India

${ }^{k}$ Institute for Space-Earth Environmental Research, Nagoya University, Nagoya 464-8601, Japan

${ }^{l}$ Institute for Cosmic Ray Research, Tokyo University, Kashiwa, Chiba 277-8582, Japan

${ }^{m}$ Indian Institute of Technology Indore, Indore 453552, India

${ }^{n}$ Graduate School of Information Sciences, Hiroshima City University, Hiroshima 731-3194, Japan 